\title{
Abordagem terapêutica da Acalásia de Esôfago: relato de caso
}

\author{
Therapeutic approach to esophageal achalasia: case reports
}

Abordaje terapéutico de la Acalasia del Esófago: informes de casos

Domires da Silva Andrade ${ }^{1 *}$, João Pedro de Resende Côrtes ${ }^{1}$, Paula Barbosa Fernandes ${ }^{1}$, Isabella Ferraz Ferreira', Paula Pitta de Resende Côrtes², Adriana Rodrigues Ferraz ${ }^{1}$.

\begin{abstract}
RESUMO
Objetivo: Relatar sobre a Acalásia de Esôfago, com diferentes resultados na esofagomanometria de alta resolução, e com desfechos terapêuticos distintos. Detalhamento dos Casos: Estudo observacional e descritivo de dois casos, em que as duas pacientes, ambas do sexo feminino, de 37 e 20 anos de idade, procuraram atendimento médico especializado, com história clínica compatível com Acalásia de Esôfago. A confirmação do diagnóstico se deu após a realização de Esofogograma Baritado, seguido por Manometria Esofágica de alta resolução, que evidenciaram diferentes classificações para essas duas pacientes. Sendo assim, foram submetidas a terapias distintas, de acordo com a melhor opção para cada circunstância, e permanecem assintomáticas até o presente momento. Considerações Finais: Conclui-se que, embora a terapêutica não atue no fator etiológico da doença, ela é fundamental para o alívio dos sintomas, e deve ser individualizada de acordo com cada paciente.
\end{abstract}

Palavras-chave: Acalasia esofágica, Manometria, Miotomia de Heller.

\begin{abstract}
Objective: Reporting on Esophageal Achalasia, with different results in high resolution esophageal manometry, and different therapeutic outcomes. Detailing of cases: Observational and descriptive study of two cases, in which the two patients, both female, 37 and 20 years old, sought specialized medical care, with a clinical history compatible with Esophageal Achalasia. The diagnosis was confirmed after the performance of a Barium Esophagogram, followed by high resolution Esophageal Manometry, which showed different classifications for these two patients. Therefore, they were submitted to different therapies, according to the best option for each circumstance, and remain asymptomatic until the present moment. Final Considerations: It is concluded that, although the therapy does not act on the etiological factor of the disease, it is fundamental for the relief of symptoms, and should be individualized according to each patient.
\end{abstract}

Keywords: Esophageal achalasia, Manometry, Heller myotomy.

\section{RESUMEN}

Objetivo: Informe sobre la Acalasia del Esófago, con distintos resultados en la manometría esofágica, y con diferentes desenlaces terapéuticos, además de realizar un repaso literario sobre la actual intervención para cada subtipo de la enfermedad. Detalles de los casos: Estudio de observacion y descripcion de dos casos, de dos pacientes, ambas del sexo feminino, 37 y 20 años de edad, buscaron atendimiento del especialista, con antecedentes clinicos compatibles con Acalasia del Esófago. El diagnóstico se confirmó después de un Esofagograma con Bario, seguido de manometría esofágica, que mostró diferentes clasificaciones para las dos pacientes. Consideraciones Finales: Se concluye que, aunque la terapia no actúe sobre el factor etiológico de la enfermedad, es fundamental para el alivio de los síntomas, y debe ser individualizada según cada paciente.

Palabras clave: Acalasia del esófago, Manometría, Miotomia de Heller.

${ }^{1}$ Universidade de Vassouras (UV), Vassouras - RJ. *E-mail: dodo-andrade@hotmail.com

2 Universidade Federal do Rio de Janeiro (UFRJ), Rio de Janeiro - RJ 


\section{INTRODUÇÃO}

A Acalasia é um distúrbio da motilidade esofágica, caracterizado por ausência de peristaltismo no corpo esofágico somada a falha de relaxamento do esfíncter esofágico inferior (ANDOLFI C e FISICHELLA PM, 2019).

Apesar de representar o transtorno motor esofagiano mais conhecido e comum, é uma desordem rara, com incidência de 1 por 100.000 pessoas por ano, e prevalência de 6-10 em 100.000 habitantes, com igual distribuição entre gêneros e aumento com a idade (JÚNIOR JEM, et al., 2017).

A maioria dos casos são representados pela etiologia idiopática, doença primária que ocorre, provavelmente, devido a fenômenos autoimunes em reposta a antígenos desconhecidos (DANTAS RO, 2013). No Brasil, estima-se que a etiologia chagásica seja responsável por $90 \%$ dos casos de pacientes com essa patologia (JÚNIOR JEM, et al., 2017; DANTAS RO, 2013).

A fisiopatologia envolve a perda irreversível de células dos plexos mioentéricos de Auerbach (neurônios inibitórios) e o de Meissner (neurônios excitatórios), estes que podem ocorrer em graus variáveis, fazendo com que a doença tenha variações na apresentação clínica e patológica (DANTAS RO, 2013).

Dentre a sintomatologia clássica, estão presentes: disfagia, que pode se apresentar no início tanto para sólido quanto para líquido (70-97\%), regurgitação (75\%) e perda ponderal (60\%), devido a menor ingestão e esvaziamento esofagiano ineficaz. Outros sintomas podem estar presentes são: dor torácica, complicações respiratórias (pneumonia por aspiração) e sitofobia (LEONARDI CJ e CURY M, 2014; DANTAS RO, 2013).

A acalásia além de proporcionar uma piora na qualidade de vida também aumenta muito o rico de desenvolver carcinoma espinocelular (CEC) em relação à população geral. O contato contínuo com a saliva, restos alimentares e proliferação de bactérias no corpo esofágico pode levar a uma esofagite crônica, displasia e finalmente ao câncer, junto à fatores de risco mais conhecidos como álcool e tabagismo são ferramentas fundamentais para o desenvolvimento desse subtipo, não tendo relação demostrada com o adenocarcinoma de esôfago (TUSTUMI F, 2018).

O escore de Eckart tem como objetivo avaliar a frequência e a intensidade dos sintomas (perda de peso, disfagia, dor torácica e regurgitação), além de estimar a gravidade da doença e a eficácia do tratamento. Os escores de 0-1 correspondem ao estágio clínico 0; 2-3 ao estágio I; 4-6 ao estágio II; e um escore $>6$ ao estágio III (LAURINO-NETO RM, et al., 2018).

O diagnóstico é feito por uma análise clínica sistemática aliada a diversos exames complementares. 0 esofagograma baritado, que consiste no primeiro passo da investigação, permite a visualização da retenção de contraste e a aplicação da escala de Resende, que avalia o grau de dilatação no estudo de esôfago contrastado, a incoordenação do trânsito e o estreitamento da transição esofagogástrica (LEONARDI CJ e CURY M, 2014; DANTAS RO, 2013).

Após o estudo de deglutição de bário, a Endoscopia Digestiva Alta (EDA) é de extrema importância, visto que permite excluir a pseudoacalásia sob consequência de doenças infiltrativas e processos neoplásicos. A esofagomanometria de alta resolução é considerada padrão ouro para o diagnóstico e determinação do subtipo de acalasia, demonstrando hipertonia e falta de relaxamento do esfíncter esofágico inferior. Devido ao elevado custo e especificidade, ela é o último exame a ser feito, geralmente já com o diagnóstico de acalásia praticamente fechado, restando somente a necessidade de determinar o subtipo (LAURINO-NETO RM, et al., 2018; JÚNIOR JEM, et al., 2017).

Embora o tratamento não atue no fator etiológico, ele é fundamental para o alívio dos sintomas. Dentre as medidas terapêuticas, estão inclusas as abordagens dietéticas, farmacológicas, endoscópicas e cirúrgicas (DANTAS RO, 2013; LOPUSHINSKY SR e URBACH DR, 2006)

O objetivo do presente estudo é relatar sobre a acalásia, com diferentes resultados na esofagomanometria de alta resolução e com desfechos terapêuticos distintos, além de realizar uma revisão da literatura sobre a terapêutica atual de cada subtipo da doença. 


\section{DETALHAMENTO DE CASO}

Este processo foi submetido ao Comitê de Ética em Pesquisa (CEP) e, de acordo as normas do CEP e documentos apresentados, o projeto atendeu à solicitação, sendo considerado aprovado, sendo o número de Aprovação do CEP: 4.227.638.

Segundo os dados do parecer, o projeto atende às solicitações da legislação, os objetivos apresentados são claros, havendo compatibilidade com a metodologia proposta, o projeto apresenta riscos significativos para os participantes, e, por fim, o projeto apresenta adequadamente; a metodologia do estudo; os critérios de inclusão e exclusão; a relação risco-benefício;

\section{Relato 1}

Paciente do gênero feminino, 37 anos, do lar, relatou ter procurado consultório de Gastroenterologia em 2015 com quadro de dor torácica e eructações excessivas. Nesta consulta, foi solicitada Endoscopia Digestiva Alta, que evidenciou Gastrite endoscópica enantematosa leve, teste da urease negativo.

Em 2019, devido às mesmas queixas, procurou outro especialista e realizou nova Endoscopia Digestiva Alta, que sugeriu acalásia com presença de megaesôfago grau II. Foi solicitada a seguir Manometria esofágica, realizada em fevereiro/2019, que confirmou o diagnóstico de acalásia. Optou-se por complementar este exame com Manometria esofágica de alta resolução, que evidenciou acalásia do subtipo II, conforme classificação de Chicago 3.0. Realizou exame de sangue com Sorologia para Doença de Chagas (Reação de Machado Guerreiro) negativa.

A seguir, a paciente foi encaminhada para avaliação com Endoscopista especializado em terapias endoscópicas, que realizou nova Endoscopia Digestiva Alta em abril/2019, cujo resultado evidenciou acalásia com repercussão funcional.

Foram realizadas nesta ocasião biópsias da cárdia e passagem de fio guia. Sob visão direta foi realizada dilatação pneumática da cárdia com balão WCAB-30 da Cook ${ }^{\mathrm{TM}}$, sendo utilizadas 3 ATM de pressão, atingindo-se o diâmetro final de $30 \mathrm{~mm}$ (Figura 1). Revisão endoscópica após a dilatação evidenciou lacerações superficiais, sem sinais de perfuração (Figura 2). Paciente assintomática até a redação do presente artigo.

Figura 1 - Endoscopia Digestiva Alta evidenciando dilatação pneumática de cárdia.

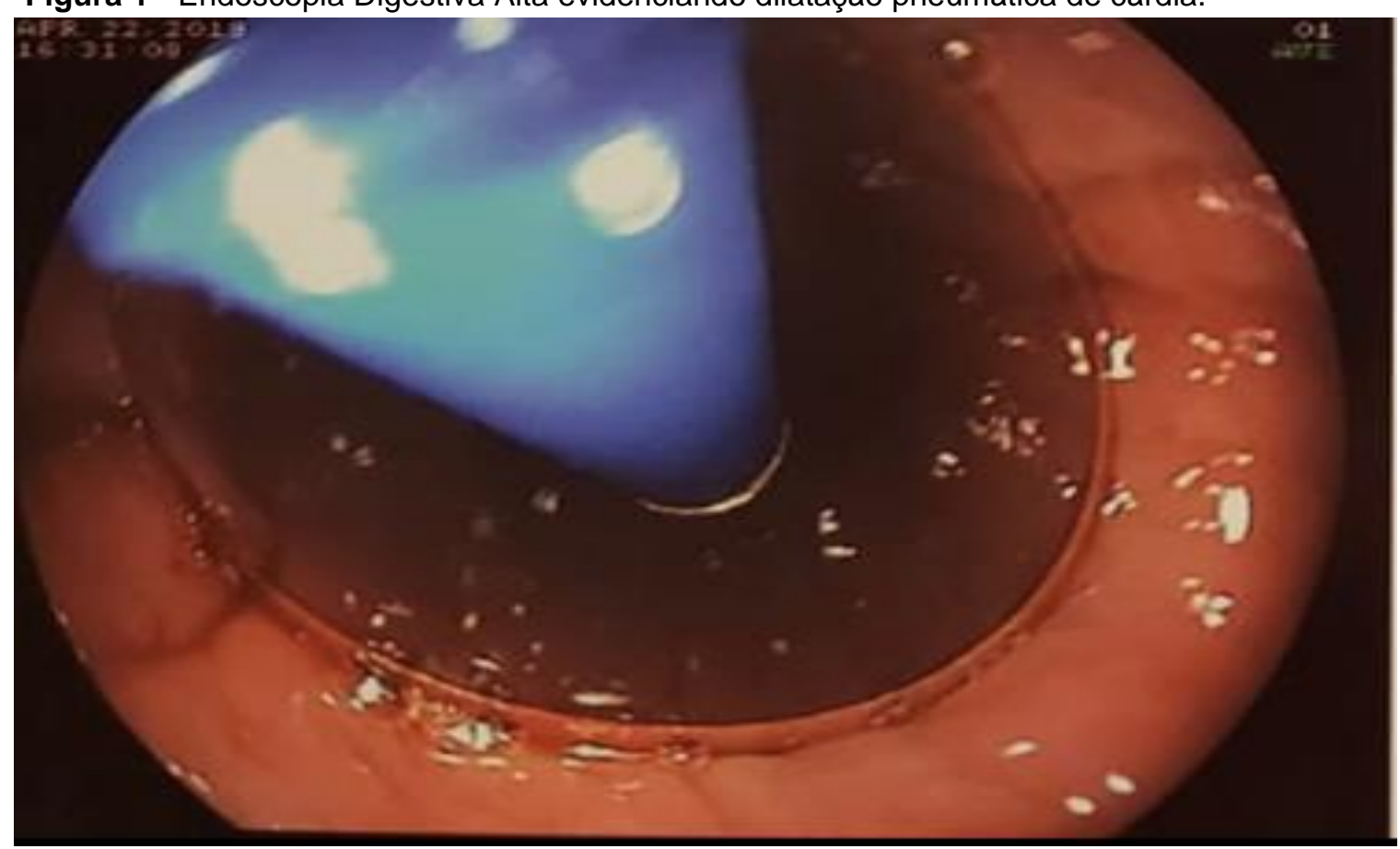

Fonte: ANDRADE DS, et al., 2020. 
Figura 2 - Revisão endoscópica após o procedimento.

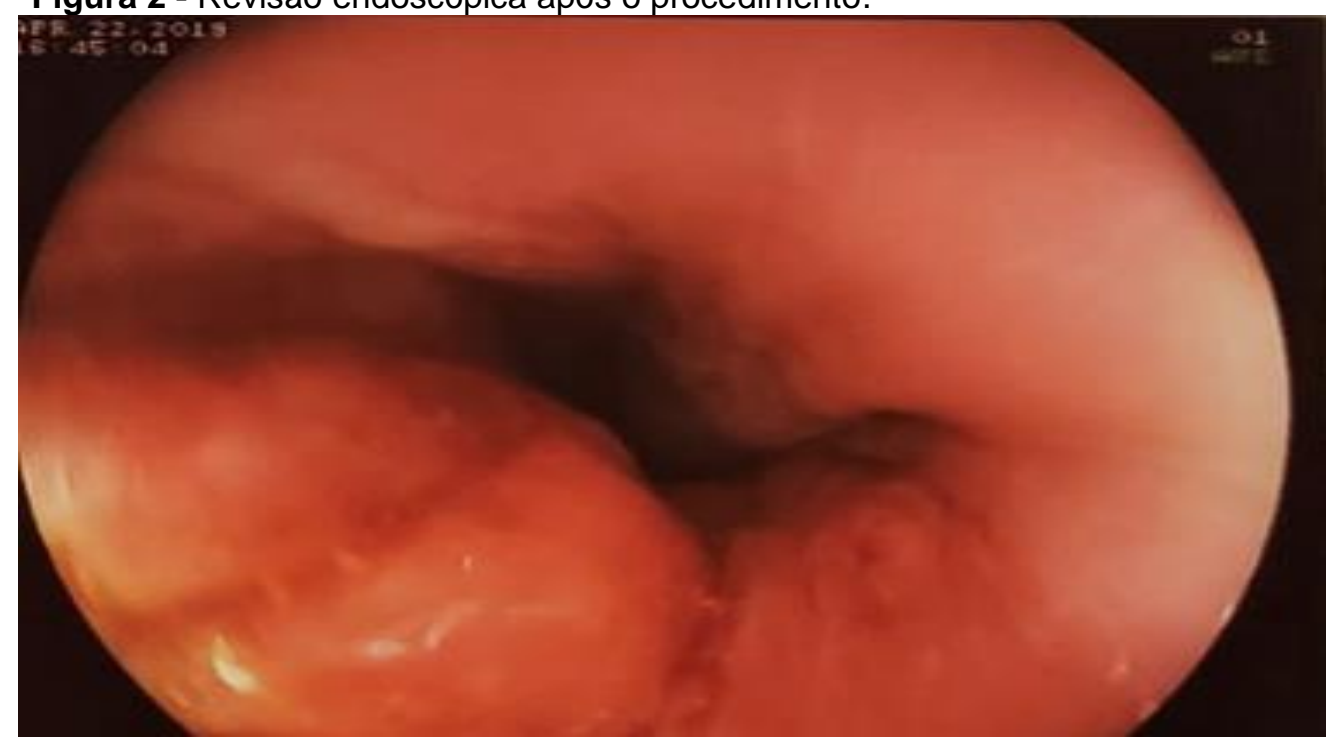

Fonte: ANDRADE DS, et al., 2020.

\section{Relato 2}

Paciente do gênero feminino, 20 anos, estudante, relatou ter procurado o setor de emergência em dez/2018, com quadro agudo de provável amigdalite. Relatou nesta consulta dificuldade para deglutir de longa data, além de emagrecimento progressivo. Já tinha realizado Endoscopia Digestiva Alta há 2 anos, normal, pela mesma queixa. Foi devidamente medicada e orientada a procurar consultório de Gastroenterologia.

$\mathrm{Na}$ consulta com especialista, foi solicitada Endoscopia digestiva alta, realizada em fev/2019 que sugeriu o diagnóstico de acalásia da cárdia com megaesôfago grau I/II, teste da urease negative (Figura 3 e Figura 4). Foi solicitada a seguir Manometria esofágica de alta resolução, realizada em março/2019, que confirmou o diagnóstico de acalásia do subtipo I, conforme classificação de Chicago 3.0. Realizou exame de sangue com Sorologia para Doença de Chagas (Reação de Machado Guerreiro) negativa.

Figura 3 - Endoscopia Digestiva Alta evidenciando calibre algo aumentado com grande quantidade de resíduos líquidos, não exibindo contrações peristálticas e com discreta tortuosidade.

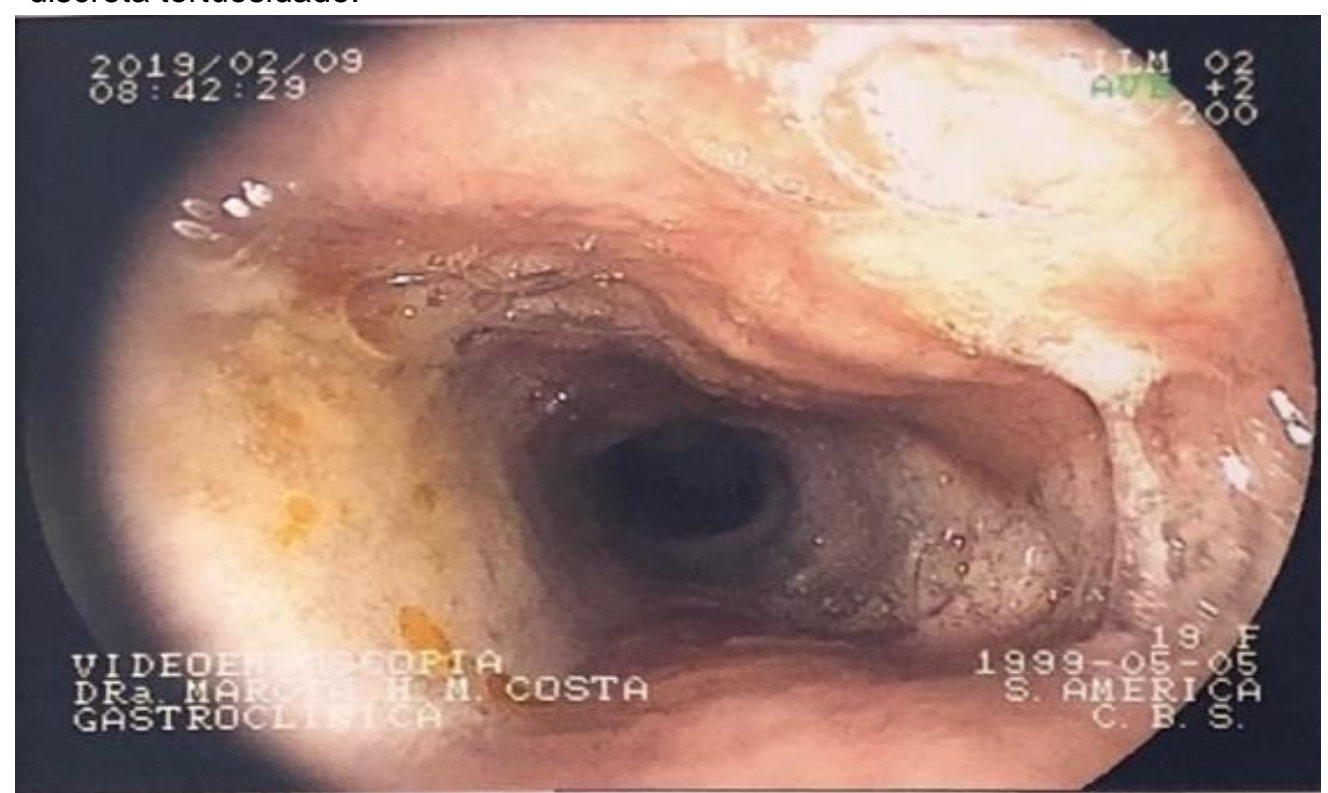

Fonte: ANDRADE DS, et al., 2020. 
Figura 4 - Endoscopia Digestiva Alta Figura com Esfíncter Esofagiano Inferior permanentemente fechado oferecendo resistência a passagem do aparelho.

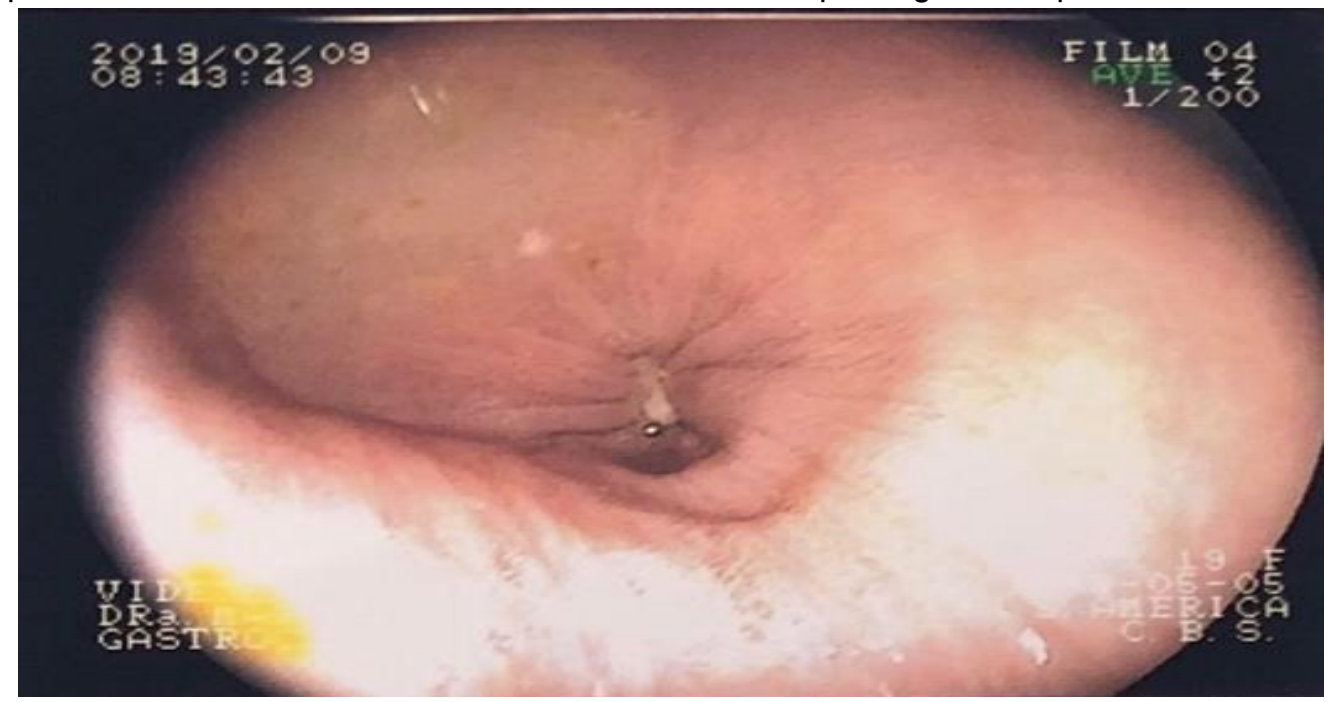

Fonte: ANDRADE DS, et al., 2020.

A seguir, a paciente foi encaminhada para avaliação com Cirurgião geral e realizou Seriografia em abril/2019 (Figura 5). O resultado evidenciou trânsito esofagiano lentificado, com esôfago dilatado com 5,0 $\mathrm{cm}$ de diâmetro na incidência AP ortostática, redução de calibre da junção esôfago gástrica com contorno regular e aspecto sugestivo de megaesôfago por acalásia. Em 19/4/2019, realizou cirurgia que consistiu na liberação da membrana frenoesofágica, esofagomiotomia de $7 \mathrm{~cm}$ a Heller e fundoplicatura anterior a dor. Paciente assintomática até a redação do presente artigo.

Figura 5 - Seriografia sugestiva de megaesôfago grau I/II por acalasia.

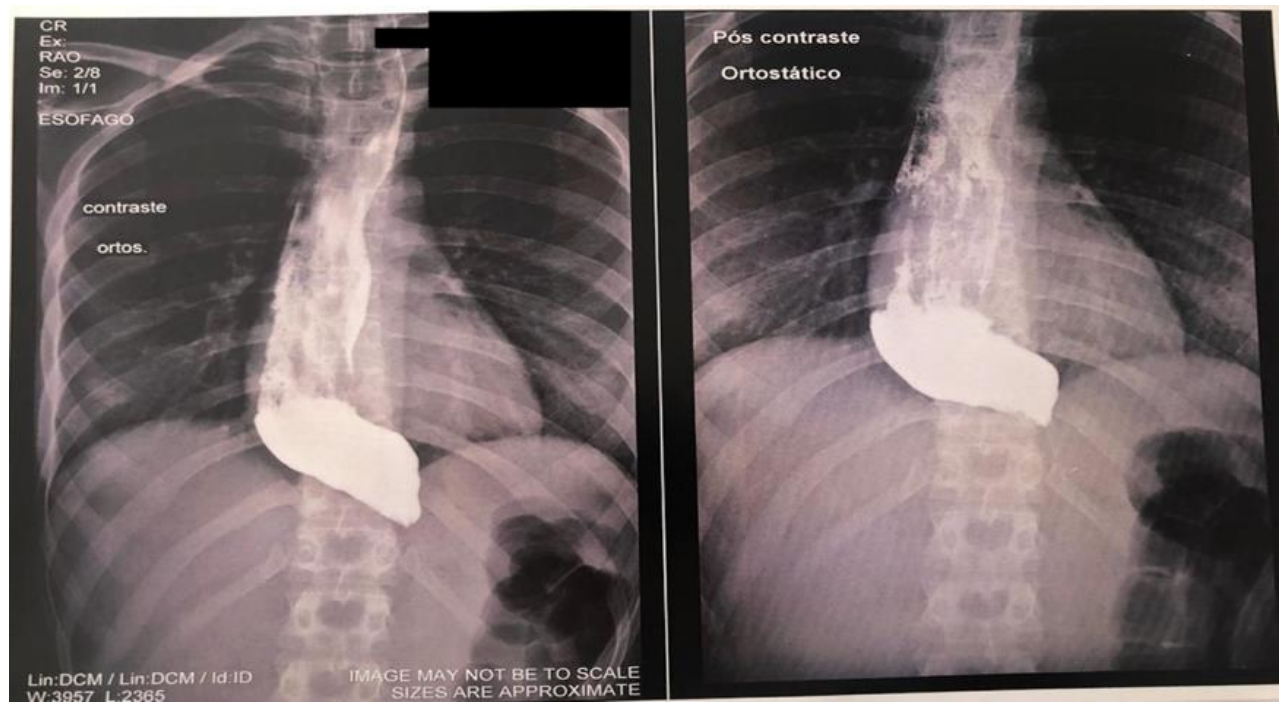

Fonte: ANDRADE DS, et al., 2020.

\section{DISCUSSÃO}

O tratamento da acalasia objetiva a diminuição do tônus do esfíncter esofagiano inferior, e a melhor opção terapêutica ainda está em discussão. Inicialmente, há preferência por tratamento medicamentoso com bloqueadores de canal de cálcio ou nitratos, porém além de possuírem curta duração, também apresentam efeitos adversos e resposta insatisfatória em $50 \%$ dos doentes. Ainda dentro das terapias menos invasivas, a toxina botulínica constitui o outro método disponível (SILVA IV, et al., 2015). 
Entretanto, se tratando de superioridade em relação aos resultados, têm-se a dilatação pneumática, a miomectomia de Heller laparoscópica (LHM) e a miotomia per-oral endoscópica (POEM) (ANDOLFI C e FISICHELLA PM, 2019; LOPUSHINSKY SR e URBACH DR, 2006).

A fim de determinar a abordagem terapêutica, há necessidade de considerar tanto a história clínica quanto os exames subsidiários. A esofagomanometria de alta resolução permite uma análise detalhada da motilidade esofagiana, possibilitando classificar a acalásia segundo a escala de Chicago (versão 3.0) em três subgrupos diferentes, de acordo com a pressurização do esôfago (Tabela 3) (CRESPIN OM, et al., 2017; HERBELLA FA, et al., 2016).

Tabela 3 - Alterações observadas na Esofagomanometria de acordo com a escala de Chicago 3.0

\begin{tabular}{cc}
\hline Tipos & $\begin{array}{c}\text { Alterações observadas } \\
\text { na Esofagomanometria }\end{array}$ \\
\hline Te acalásia & Ausência de Pressurização esofágica e de contratilidade \\
Tipo II & Ausência de peristalse e $\geq 20 \%$ de Pressurização Panesofágica \\
Tipo III & $\geq 20 \%$ de contrações prematuras (latência distal $<4.5 \mathrm{seg}$ )
\end{tabular}

Fonte: ANDRADE DS, et al., 2020. Dados extraídos de LAURINO-NETO RM, et al., 2018.

A metanálise de Andolfi C e Fisichella PM (2019), demonstrou que na acalásia tipo I há uma taxa de sucesso de $61 \%$ para dilatação pneumática, $81 \%$ para LHM e $95 \%$ para a POEM. Na tipo II, $84 \%$ para dilatação, 92\% para LHM e 97\% para POEM, não havendo diferença nas chances de sucesso significativas entre a POEM e a LHM (OR 1.31, 0.48 para 3.55; P =0.591). Enquanto na tipo III é de $31 \%$ para dilatação, $71 \%$ para LHM e 93\% para POEM (ANDOLFI C e FISICHELLA PM, 2019; CRESPIN OM, et al., 2017; LAURINO-NETO RM, et al., 2018).

A acalásia tipo II apresenta melhor resposta pós-operatória, independentemente do método terapêutico realizado, em comparação ao tipo I e III. A melhor capacidade da POEM de estender a miotomia proximalmente para o esôfago torácico pode explicar seus bons resultados, principalmente no subtipo III, apesar de não ser o método preferencialmente empregado por sua baixa disponibilidade, elevado custo e poucos estudos prospectivos observacionais que o apoiem (ANDOLFI C e FISICHELLA PM, 2019; CRESPIN OM, et al., 2017; LAURINO-NETO RM, et al., 2018; VON RENTELN D, et al., 2012).

O advento do NOTES (Cirurgia Endoscópica Translumial por Orifícios) possibilitou um lugar de destaque para a POEM dentre as atuais abordagens terapêuticas. De acordo com o consenso de Kagoshima para acalasia, recomenda-se que a miotomia se estenda $4-5 \mathrm{~cm}$ no esôfago e $2-3 \mathrm{~cm}$ no estômago, valores inferiores a esses podem resultar em uma maior taxa de recidiva, porém, o comprimento ideal para POEM ainda é discutível (LEONARDI CJ e CURY M, 2014).

Um estudo randomizado demostrou sucesso terapêutico de 80-97\% após 12 meses que é definido como escore de ECKARDT $\leq 3$. A técnica, mesmo sendo mais invasiva que a dilatação, apresenta risco de complicações graves inferior à última, sendo uma boa opção para pacientes refratários ao tratamento por balão. O procedimento apresenta como maior desvantagem, a alta taxa de doença do refluxo gastroesofágico, que no estudo foi de $49 \%$ dos pacientes em 1 ano (PONDS FA, et al., 2019).

Cita-se também como desvantagem, a tecnologia de difícil acesso e o maior custo do procedimento, mas alguns autores relatam que a nova técnica possui um melhor custo-utilidade em relação à miotomia de Heller (CONTE TM, 2019).

Dentre as principais complicações da POEM pode-se citar: pneumoperitônio, enfisema subcutâneo, atelectasia, pneumomediastino, pneumorretroperitônio, derrame pleural e sangramento. Possuem baixa incidência, tendo em vista a disponibilidade do método concentrada em centros especializados, onde a técnica, aperfeiçoamento e aprimoramento são desenvolvidos (REN Z, et al., 2012; ARANTES V, et al., 2013).

A dilatação por balão é um processo minimamente invasivo e o mais utilizado em todo mundo, principalmente em países em desenvolvimento, devido ao seu custo inferior a POEM e a LHM. A modalidade objetiva a distensão do corpo esofágico e a desorganização das fibras musculares na topografia do esfíncter esofágico inferior (PONDS FA, et al., 2019; GUPTA SJ, et al., 2017). 
O gradiente de pressão deve ser adequado tanto para se obter alívio sintomático, quanto para evitar risco de perfuração esofágica, sua principal complicação. Há necessidade de relatar que o alívio sintomático é superior a 78\%, porém $1 / 3$ reportam recorrência de sintomas após 4 a 6 anos da intervenção, sendo necessárias novas dilatações com diferentes dimensões ou outros métodos terapêuticos (GUPTA SJ, et al., 2017; MARKAR SR, et al., 2018).

O sucesso da dilatação depende da experiência profissional e da inexistência de procedimentos cirúrgicos prévios, devido ao risco de perfuração da mucosa (MARKAR SR, et al., 2018; LYNCH KL, et al., 2012). O subtipo II, a idade maior que 45 anos, o sexo feminino e uma pressão do esfíncter esofágico inferior, inferior a $10 \mathrm{mmHg}$ após procedimento, são considerados fatores preditivos favoráveis para esse procedimento (GUPTA SJ, et al., 2017; LYNCH KL, et al., 2012).

A LHM consiste na secção longitudinal da musculatura esofagiana na topografia do esfíncter esofágico inferior visando diminuir sua pressão. Atualmente, a Heller modificada resume-se em uma miotomia abrangendo $6 \mathrm{~cm}$ do esôfago e $2 \mathrm{~cm}$ abaixo da junção esôfagogástrica, com acréscimo de uma válvula parcial anti-refluxo (BANIYA R, et al., 2017; SILVA CM, et al., 2011).

A técnica isolada está associada a refluxo em $50-60 \%$ dos pacientes, sendo assim a maioria dos autores recomenda associar técnicas antirrefluxo ao procedimento, como a fundoplicatura de Toupet (MARKAR SR, et al., 2018; BANIYA R, et al., 2017; SILVA CM, et al., 2011).

Desde 1991, a via laparoscópica para tratamento do megaesôfago vem sendo adotada, demonstrando maiores benefícios para o paciente, devido ao menor tempo de internação hospitalar e de retorno as atividades (SILVA CM, et al., 2011).

Inicialmente, a maioria dos cirurgiões optam por tratamento cirúrgico conservador, no qual é feito a secção incompleta da musculatura esofágica associada à fundoplicatura parcial, gerando resultados satisfatórios em 64 a $87 \%$ dos pacientes e baixa morbimortalidade quando comparada a esofagectomia.

Desse modo, opta-se pela secção esofagiana radical em casos avançados da doença, presença de lesões pré-malignas e falha no tratamento cirúrgico conservador, tendo em vista sua morbidade de $35 \%$, que advém principalmente de complicações pleuropulmonares, podendo chegar a 10\% de mortalidade (LEONARDI CJ e CURY M, 2014).

Atualmente, a POEM possui maior taxa de sucesso em todos os subtipos, em especial o III, porém há pouca disponibilidade e restrição de estudos controlados e randomizados que corroborem seu uso. Alternativamente, é possível concluir que a LHM se mostra superior a longo prazo, em todas as categorias, quando comparada à dilatação pneumática.

Devido à falta de consenso, faz-se necessário um olhar multidisciplinar, levando em consideração os diversos aspectos envolvidos, tanto intrínsecos, quanto extrínsecos ao paciente, de modo a estabelecer um tratamento individualizado, que alcançará melhora na qualidade de vida e uma melhor relação custobenefício.

\section{REFERÊNCIAS}

1. ANDOLFI C, FISICHELLA PM. Meta-analysis of clinical outcome after treatment for achalasia based on manometric subtypes. Br J Surg, 2019 Mar; 106(4):332-341.

2. ARANTES V, et al. Standardized endoscopic submucosal tunnel dissection for management of early esophageal tumors (with video). Gastrointest Endosc., 2013; 78(6): 946-52.

3. BANIYA R, et al. Laparoscopic esophageal myotomy versus pneumatic dilation in the treatment of idiopathic achalasia: a meta-analysis of randomized controlled trials. Clin Exp Gastroenterol, 2017; 10: 241-248.

4. CONTE TM. Custo-utilidade da miotomia endoscópica peroral (POEM) comparada a miotomia laparoscópica a Heller associada à fundoplicatura em pacientes portadores de acalasia. Tese (Doutorado em Ciências em Gastroenterologia) - Faculdade de Medicina. Universidade de São Paulo, São Paulo, 2019; 133p.

5. CRESPIN OM, et al. The relationship between manometric subtype and outcomes of surgical treatment for patients with achalasia: Achalasia: manometric subtypes. Surg Endosc., 2017; 31(12): 5066-5075.

6. DANTAS RO. Comparação entre acalásia idiopática e acalásia conseqüente à doença de Chagas: revisão de publicações sobre o tema. Arq. Gastroenterol., 2013; 40(2): 126-130. 
7. GUPTA SJ, et al. Pneumatic Balloon Dilatation for Achalasia Cardia: Outcome, Complications, Success, and Longterm Follow-up. Euroasian J Hepato- Gastroenterol, 2017; 7(2): 138-141.

8. HERBELLA FA, et al. Apresentação em imagens da Classificação de Chicago versão 3.0 das doenças da motilidade esofagiana. Einstein, 2016; 14(3): 439-442.

9. JÚNIOR JEM, et al. Tratamento cirúrgico de acalasia pela realização de esofagocardiomiotomia com fundoplicatura videolaparoscópica à Heller-Pinotti em paciente com megaesôfago grau IV: Relato de caso. Rev Med Minas Gerais, 2017; 27: e-1884.

10. LAURINO-NETO RM, et al. Avaliação diagnóstica da acalasia do esôfago: dos sintomas à classificação de Chicago. ABCD Arq Bras Cir Dig, 2018; 31(2): e1376.

11. LEONARDI CJ, CURY M. Estudo prospectivo para tratamento de acalasia pela técnica de miotomia endoscópica POEM (Peroral Endoscopic Myotomy). GED gastroenterol. endosc. dig., 2014; 33(1): 7-13.

12. LOPUSHINSKY SR, URBACH DR. Pneumatic Dilatation and Surgical Myotomy for Achalasia. JAMA, 2006; 296(18): 2227-2233.

13. $\mathrm{LYNCH} \mathrm{KL}$, et al. Major complications of pneumatic dilation and Heller myotomy for achalasia: single-center experience and systematic review of the literature. Am J Gastroenterol., 2012; 107(12): 1817-25.

14. MARKAR SR, et al. Population-based cohort study of surgical myotomy and pneumatic dilatation as primary interventions for oesophageal achalasia. Br J Surg, 2018; 105(8): 1028-1035.

15. PONDS FA, et al. Effect of Peroral Endoscopic Myotomy vs Pneumatic Dilation on Symptom Severity and Treatment Outcomes Among Treatment-Naive Patients with Achalasia: A Randomized Clinical Trial. JAMA, 2019; 322(2): 134144.

16. REN Z, et al. Perioperative management and treatment for complications during and after peroral endoscopic myotomy (POEM) for esophageal achalasia (EA) (data from 119 cases). Surg Endosc., 2012; 26(11): 3267-3272.

17. SILVA CM, et al. Cardiomiotomia com fundoplicatura videolaparoscópica no tratamento do megaesôfago não avançado. ABCD, arq. bras. cir. dig., 2011; 24(3): 195-199.

18. SILVA IV, et al. Acalásia na adolescência: dois casos. Nascer e Crescer, 2015; 24(1): 24-29.

19. TUSTUMI F. Carcinoma de esôfago em pacientes com acalasia: revisão sistemática e metanálise. Dissertação (Mestrado em Ciências) - Faculdade de Medicina. Universidade de São Paulo, São Paulo, 2018; 63 p.

20. VON RENTELN D, et al. Peroral endoscopic myotomy for the treatment of achalasia: a prospective single center study. Am J Gastroenterol., 2012; 107(3): 411-417. 